\title{
Evidences of the Low Implication of Mosquitoes in the Transmission of Mycobacterium ulcerans, the Causative Agent of Buruli Ulcer
}

\author{
Rousseau Djouaka, ${ }^{1}$ Francis Zeukeng, ${ }^{1,2}$ Jude Daiga Bigoga, ${ }^{2}$ David N'golo Coulibaly, ${ }^{3}$ \\ Genevieve Tchigossou, ${ }^{1,4}$ Romaric Akoton, ${ }^{1,4}$ Sylla Aboubacar, ${ }^{3}$ \\ Sodjinin Jean-Eudes Tchebe, ${ }^{4}$ Clavella Nantcho Nguepdjo, ${ }^{2}$ Razack Adeoti, ${ }^{1}$ \\ Innocent Djegbe, ${ }^{1}$ Manuele Tamo, ${ }^{1}$ Wilfred Fon Mbacham, \\ Solange E. Kakou-Ngazoa, ${ }^{3}$ and Anthony Ablordey ${ }^{5}$
}

${ }^{1}$ AgroEcoHealth Platform, International Institute of Tropical Agriculture (IITA), 08 P.O. Box 0932, Tri-Postal, Cotonou, Benin ${ }^{2}$ Faculty of Science, Department of Biochemistry, University of Yaoundé I, P.O. Box 812, Yaoundé, Cameroon

${ }^{3}$ Department of Technics and Technology, Platform of Molecular Biology, Pasteur Institute Abidjan, P.O. Box 490 Abidjan 01, Abidjan, Côte d'Ivoire

${ }^{4}$ Faculty of Science and Techniques, University of Abomey-Calavi, P.O. Box 526, Abomey-Calavi, Benin

${ }^{5}$ Department of Bacteriology, Noguchi Memorial Institute for Medical Research, University of Ghana, P.O. Box 581, Legon, Accra, Ghana

Correspondence should be addressed to Francis Zeukeng; zeusfranck07@yahoo.com

Received 13 May 2017; Revised 8 July 2017; Accepted 17 July 2017; Published 28 August 2017

Academic Editor: Maria De Francesco

Copyright (C) 2017 Rousseau Djouaka et al. This is an open access article distributed under the Creative Commons Attribution License, which permits unrestricted use, distribution, and reproduction in any medium, provided the original work is properly cited.

Background. Buruli ulcer (BU) continues to be a serious public health threat in wet tropical regions and the mode of transmission of its etiological agent, Mycobacterium ulcerans $(M U)$, remains poorly understood. In this study, mosquito species collected in endemic villages in Benin were screened for the presence of $M U$. In addition, the ability of mosquitoes larvae to pick up $M U$ from their environment and remain colonized through the larval developmental stages to the adult stage was investigated. Methods. 7,218 adults and larvae mosquitoes were sampled from endemic and nonendemic villages and screened for MU DNA targets (IS2404, IS2606, and KR-B) using qPCR. Results. $M U$ was not detected in any of the field collected samples. Additional studies of artificially infected larvae of Anopheles kisumu with $M U$ strains revealed that mosquitoes larvae are able to ingest and host $M U$ during L1, L2, L3, and L4 developmental stages. However, we noticed an absence of these bacteria at both pupae and adult stages, certainly revealing the low ability of infected or colonized mosquitoes to vertically transmit $M U$ to their offspring. Conclusion. The overall findings highlight the low implication of mosquitoes as biological vectors in the transmission cycle of $M U$ from the risk environments to humans.

\section{Introduction}

Buruli ulcer (BU) is a neglected emerging disease that has recently been reported in some countries as the second most frequent mycobacterial disease in humans after tuberculosis [1-3]. BU continues to be one of the most debilitating cutaneous diseases causing significant morbidity. The disease is characterized by severe subcutaneous necrotic lesions that lead to chronic opened sores and ulcerations, ultimately affecting the bone in extreme cases [4]. Mycolactone, a secreted exotoxin, is the only virulence factor identified to date for Mycobacterium ulcerans (MU) [5].

During the last two decades, there has been a reemergence of $\mathrm{BU}$ across diverse regions of the world $[3,6,7]$. Its prevalence has increased and currently is seen in over 33 countries worldwide [8]. Although the distribution of $\mathrm{BU}$ is 
global and affects people of all ages, the burden of this disease is most severe in West and Central Africa, as well as some parts of Australia $[1,9,10]$. More than 30,000 cases of BU have been reported in Africa over the last decade and the West African region accounts for more than $67 \%$ of the reported cases [6].

The environmental pathogen Mycobacterium ulcerans (MU) is the etiologic agent of Buruli ulcer [11]. Merritt et al. [1] provided a series of hierarchical criteria analogous to Koch's postulates and/or the Bradford Hill guidelines emphasizing epidemiological/ecological association and the use of logical inference for establishing cause and effect in biological disease transmission. They further discussed the application of this process to indictment of insect vectors for transmission of $M U$. However, the mode of transmission of $M U$ from the risk environments to humans remains unknown and its reservoirs in the environment are still being uncovered [1]. The direct transmission of $M U$ from humanto-human is extremely rare and cases usually occur in proximity to slow moving or stagnant bodies of water and among rural and economically deprived populations [12-18]. Recent studies in Australia have demonstrated that mosquitoes may be potential reservoirs or vectors of BU [7, 19-23]. Similarly, a recent study conducted in an endemic area of Cameroon revealed the presence of $M U$ molecular markers in hematophagous families of insects like Culicidae (mosquito's family), Ceratopogonidae, and Psychodidae [24]. However, a similar study in an endemic area of Benin did not detect $M U$ molecular markers in mosquito species [25]. An experimental laboratory study conducted by Wallace et al. [26] also failed to confirm the implication of mosquitoes as biological vectors in the transmission of BU. These recent studies highlight controversial concerns whether mosquitoes actually play a role in the transmission dynamics of BU. Mosquitoes are the most important group of insects involved in the spread of human and animal diseases [27]. One hypothesis is that they could transmit $M U$ to humans. However, there is no scientific or historic precedent for mosquitoes transmitting a bacterium to host in any disease system, either directly or mechanically [1]. In the vector ecology, they may serve as biological vectors and hosts for pathogen replication, or mechanical vectors carrying organisms from hosts to hosts without serving as a site of replication [26, 27]. This last hypothesis has recently been reinforced by Wallace et al. [28] who reported a biologically plausible mechanical transmission mode of BU via natural or anthropogenic skin punctures (trauma). These authors further highlighted that a significant low quantity of $M U$ delivered beneath the skin surface of animal (BALB/C mice) by a minor injury created by mosquitoes might cause BU in return [28]. Previously in 1974, Meyers et al. [29] reported that skin trauma could be an important mode of transmitting $M U$ infections or of introducing $M U$ into the dermis of subcutaneous tissue from superficially contaminated skin. However, Williamson et al. [30] recently established that abrasions (trauma) of the skin in Guinea pig models and subsequent application of $M U$ are not sufficient enough to cause an ulcer. Mosquitoes contamination or colonization by $M U$ remains an event which has only been reported in Australia and which could vary according to mosquito species. As BU infections occur in humid areas of Africa where high densities of mosquito species are recorded, there is a need to further investigate whether they could be involved in the transmission cycle of BU in African settings and more specifically in Benin.

In this study, we tested the hypothesis of the implication of mosquito species in the transmission of $M U$ in an endemic area of Sedje-Denou in the Southern Benin. We further evaluated whether mosquitoes could pick $M U$ bacteria from water breeding sites during larval developmental stages leading to colonization and whether colonization continues into the adult stage where they become infective to humans (vertical transmission of $M U$ by mosquitoes). Based on these assumptions, we screened wild mosquitoes populations collected from three endemic villages found in Sedje-Denou for molecular targets of $M U$. Coupled to this field based activity, we also investigated the potential for vertical transmission of $M U$ within mosquitoes populations using the laboratory strain Anopheles kisumu.

\section{Methods}

2.1. Ethical Considerations. This research which was mainly laboratory based received administrative clearance from the International Institute of Tropical Agriculture (IITA). In addition, the community consent was obtained prior to mosquitoes sampling in the three villages of Sedje-Denou.

2.2. Study Area. This study was carried out in three endemic communities (Agbahounsou, Agodenou, and Agongbo) of Sedje-Denou $\left(6^{\circ} 32^{\prime} \mathrm{N}\right.$ and $\left.2^{\circ} 13^{\prime} \mathrm{E}\right)$ in the Southern Benin (Figure 1). One nonendemic village, Tanongou $\left(10^{\circ} 48^{\prime} \mathrm{N}\right.$ and $1^{\circ} 26^{\prime} \mathrm{E}$ ), in the Northern Benin was selected as a negative control village for data comparison. Sedje-Denou (also named Sedje) is located in the Commune of Ze which is the second most endemic locality in Benin with a reported prevalence of 450 cases of BU per 100,000 inhabitants [14]. The presence of rivers and wetlands make this locality an appropriate environment for BU. According to Wagner et al. [31], drainage basins as well as forest land cover with variable wetness patterns are prolific for the growth of MU and associated with higher BU disease prevalence rates. These patterns could also influence the distribution and abundance of vectors, or mediating vector-human interactions. The climate at Sedje is a subequatorial type with two discontinuous dry and wet seasons. The annual average rainfall measures $1,000 \mathrm{~mm}$ with an annual average temperature of $24^{\circ} \mathrm{C}$ and a mean altitude of $20 \mathrm{~m}$. The population of 5,496 inhabitants are distributed into six different villages. Sedje is a rural area where agricultural works being the predominant occupation could contribute to increased exposure to $M U$ due to the close spatial proximity with the risk environments [31].

Tanongou is also a rural locality under the Department of Atakora in Northern Benin (Figure 1). This village is administratively subdivided into two close villages named Tanongou 1 and Tanongou 2. BU epidemiological data in Benin show that this locality is a nonendemic area for the disease. The climate is a wet Sudanese type with one long dry season (November to May) and a short rainy season (June to October). This region 


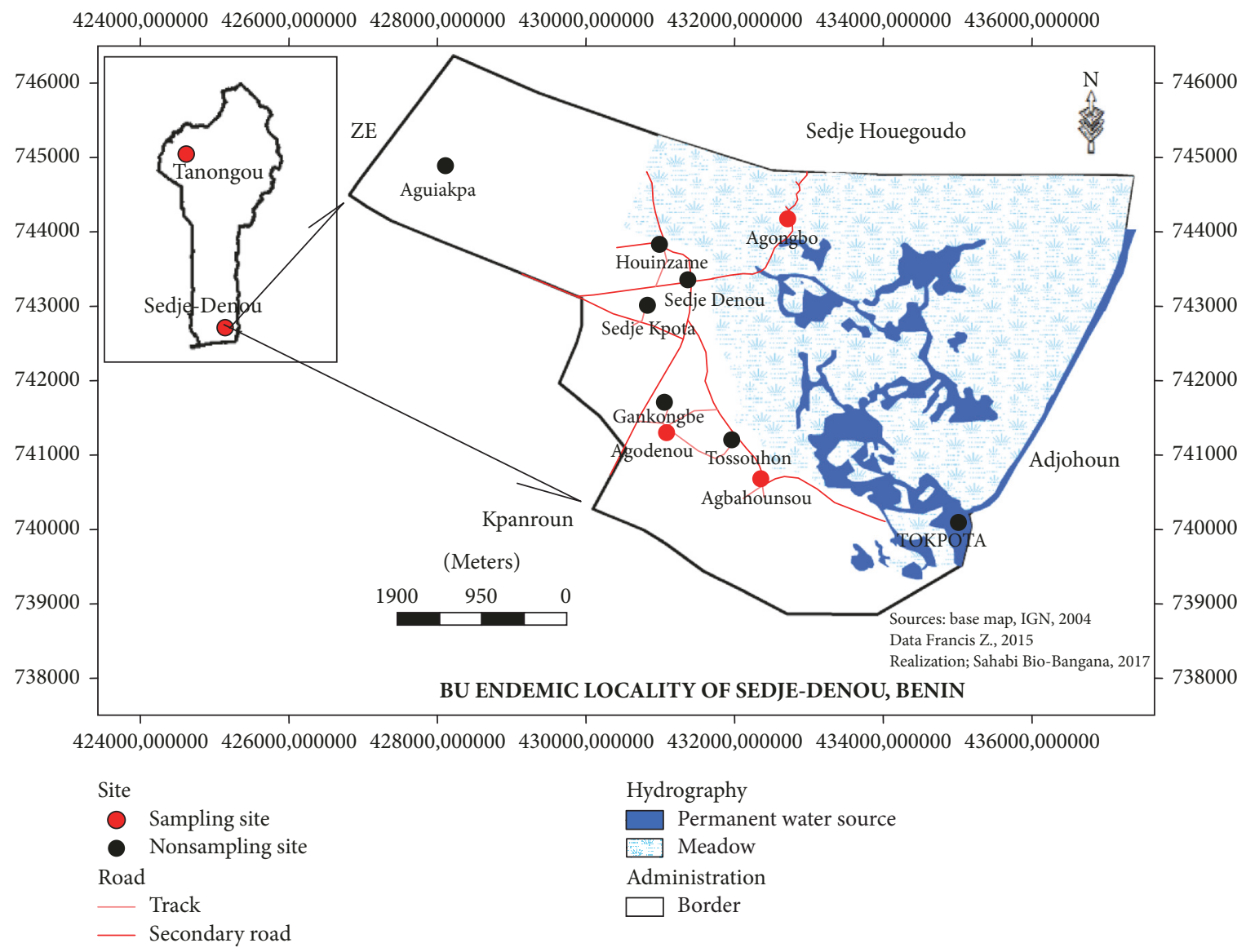

Figure 1: Location map of study sites in the Buruli ulcer endemic area in Southern Benin and the nonendemic area in Northern Benin.

is dominated by hills of up to $800 \mathrm{~m}$ of altitude and several small water bodies, which makes the region colder and relatively wet. Annual rainfall ranges from 1200 to $1300 \mathrm{~mm}$ per year, the vegetation is partially of wet savanna type, and the temperature in this part of the country ranges between 23 and $31^{\circ} \mathrm{C}$. Agriculture is the prominent activity of the region.

\subsection{Sampling of Mosquito Species in BU Endemic and Nonendemic Areas}

2.3.1. Sampling of Adult Mosquitoes. Field surveys for mosquitoes collections were conducted during rainy seasons at the 3 villages of Sedje-Denou from 2014 to 2016. Similarly, mosquitoes samples were collected at Tanongou (Tanongou $1+$ Tanongou 2) during rainy seasons as well. Adult mosquitoes were caught indoors using insecticide spraying technique which is one of the effective methods for collecting indoors resting mosquitoes [32]. Mosquitoes were harvested about twenty minutes after house spraying. They were safely transferred into Petri dishes labeled with room/house references and were taken to the laboratory. In the laboratory, each mosquitoes sample was morphologically identified using Edward identification keys [33]. Mosquitoes were identified to genus and to species. No molecular test was performed for mosquito identification. For each identified species (Anopheles gambiae s.l., Culex quinquefasciatus, Mansonia africana, and Aedes aegypti), pools of 10 mosquitoes each were prepared and kept at $-20^{\circ} \mathrm{C}$ in Eppendorf tubes filled with silica gel. Mosquitoes from Tanongou 1 and Tanongou 2 were pooled and considered as from a single control village of Tanongou.

2.3.2. Sampling of Mosquito Larvae. Mosquito larvae were collected from temporal, semipermanent, and permanent breeding areas using the WHO protocol [34]. Collected larvae were transported to the laboratory where they were morphologically identified and pooled as were the adults. Larvae pools were prepared and stored at $-20^{\circ} \mathrm{C}$ in $70 \%$ alcohol.

\subsection{Molecular Identification of MU in Mosquitoes Samples}

2.4.1. Extraction of Genomic DNA. Genomic DNA was extracted from a total of 721 pools of mosquitoes samples (adult and larvae) using the phenol/chloroform extraction method described by Sambrook and Russell [35]. Several types of controls were put in place to guide against false positive and negative results. To reduce cross-contaminations, extractions were conducted in batches of 10 pools and the 10 pools completely processed (extraction and PCRs) before moving back to a new set of extractions. Negative controls 
(nuclease-free water, Sigma-Aldrich) were added at a frequency of $10 \%$ (1 control per batch of extraction) to monitor potential cross-contaminations. Pooled mosquitoes samples were ground using an electric grinder in sterile $100 \mu \mathrm{l} 1 \mathrm{x}$ PBS and the homogenates were suspended in $300 \mu$ l preheated lysis buffer made of $5 \mathrm{M} \mathrm{NaCl}, 0.5 \mathrm{M}$ EDTA, $1 \mathrm{M}$ Tris- $\mathrm{HCl}$ ( $\mathrm{pH}$ 8.0), 10\% SDS, and proteinase K (Qiagen, Hilden). The mixture was heated at $60^{\circ} \mathrm{C}$ for one hour and DNA extracted with phenol/chloroform/isoamyl acid in the ratio $25: 24: 1$. This was briefly mixed by a pulse vortex and centrifuged for $2 \mathrm{~min}$ at $13,000 \mathrm{~g}$. The DNA was precipitated by adding 2 volumes of pure ethanol and the mixture was incubated for 2 hours and centrifuged $10 \mathrm{~min}$ at $13,000 \mathrm{~g}$. The DNA was washed by $70 \%$ cold ethanol, dried $20 \mathrm{~min}$ at room temperature, and eluted in $50 \mu \mathrm{l}$ of nuclease-free water (Sigma-Aldrich).

2.4.2. Detection of MU DNA in Mosquitoes Samples Using TaqMan qPCRs. The TaqMan IS2404 qPCR analysis described by Fyfe et al. [36] was performed on extracted mosquitoes DNA samples to detect Mycobacterium DNA in these samples. A total of 404 pools of DNA samples from adult mosquitoes and a total of 317 pools of DNA samples from mosquitoes larvae from the 4 villages ( 3 endemic villages and one control village) were subjected to PCR analysis for detecting the presence of $M U$ in these wild mosquitoes populations. Briefly, $2.5 \mu \mathrm{l}$ of the DNA extract was amplified in $12.5 \mu \mathrm{l}$ PCR mixture using the SensiMix buffer system (BioLine). Each reaction mixture contained $7.5 \mu$ l SensiMix (2x SensiMix II probe, No-Rox Mix, BioLine), 0.9 $\mu \mathrm{M}$ IS2404 primer pair, $0.25 \mu \mathrm{M}$ IS2404 probe, a reference Rox dye (Rox Passive Reference Dye, Bio-Rad), and sterile nuclease-free water (Sigma-Aldrich). One positive control ( $M U$ Agy99 DNA) as well as a no-template negative control (nucleasefree water, Sigma-Aldrich) was used to guide this experiment against false positive and negative results. The amplification process was performed in the Mx3500P automate (MxPro Agilent Technologies, Stratagene Mx3500P) under the following cycling conditions: $50^{\circ} \mathrm{C}$ for $2 \mathrm{~min}, 95^{\circ} \mathrm{C}$ for $10 \mathrm{~min}, 40$ cycles of $95^{\circ} \mathrm{C}$ for $15 \mathrm{~s}$, and $60^{\circ} \mathrm{C}$ for $1 \mathrm{~min}$. Negative samples to IS2404 were diluted $1 / 10$ and resubmitted to molecular analyses for the detection of PCR inhibitors. In addition to the screening of the IS2404 target, other quantitative real time PCR IS2606/KR multiplex assays were performed on IS2404-positive samples to screen the presence of Mycobacterium conservative insertion sequence 2606 (IS2606) and the Ketoreductase B $(K R-B)$ domain of the mycolactone polyketide synthase gene of $M U$ plasmid (pMUM001) [36]. QPCR mixtures here contained $1 \mu \mathrm{l}$ of DNA template, $0.9 \mu \mathrm{M}$ of each primer, $0.25 \mu \mathrm{M}$ of each probe, $12.5 \mu \mathrm{l}$ of the SensiMix buffer system (2x SensiMix II probe, No-Rox Mix, BioLine), and nuclease-free water (Sigma-Aldrich) in a total volume of $25 \mu \mathrm{l}$. Amplification and detection conditions were performed as described above.

2.5. Investigations on the Capability of Mosquitoes to Pick and Host MU Bacteria from Larval to Adult Stages (Vertical Transmission of $M U$ in Mosquitoes). This experiment was carried out in the insectary of the AgroEcoHealth Platform of the International Institute of Tropical Agriculture (IITABenin). The laboratory strain Anopheles gambiae kisumu and $M U$ strain isolates were used in this experiment.

2.5.1. The Mosquitoes Strain Anopheles kisumu. Anopheles kisumu is a reference laboratory strain originating from the Kisumu region in Western Kenya. This strain is commonly used in standardization experiments and is well maintained in most malaria entomology research laboratories.

2.5.2. The Bacterial Strain Mycobacterium ulcerans Agy99. Mycobacterium ulcerans Agy99 (MU Agy99) is a wellcharacterized Ghanaian human isolate obtained from the Department of Bacteriology at the Noguchi Memorial Institute for Medical Research (NMIMR, Ghana). Agy99 is a reference $M U$ strain with a sequenced genome [37].

2.5.3. Experimental Infection of Mosquitoes Larvae with Mycobacterium ulcerans and Monitoring of Infected Larvae. Mosquitoes larvae were infected by ingestion of $M U$ contaminated Tetramin ${ }^{\circledR}$ Baby Fish Food (Charterhouse Aquatics, London, UK). The infection protocol was adapted from Wallace et al. [26]. Prior to infection, the preserved stock of $M U$ strain was diluted in $1 \mathrm{X}$ PBS and vortexed $5 \mathrm{~min}$.

(1) Experimental Infection of Mosquitoes Larvae with $M U$. Six groups (4 tests and 2 controls) of 100 eggs of An. kisumu each were distributed for rearing into labeled plastic bowls containing $250 \mathrm{ml}$ sterile water. Prior to introducing eggs into bowls, the breeding/rearing water in test groups received $80 \mathrm{mg}$ of Tetramin Baby Fish Food (Charterhouse Aquatics, London, UK) contaminated with $100 \mu$ l of $M U$ (2.0 $10^{5} \mathrm{CFU} / \mathrm{ml}$ ). The control groups (2 bowls) were prepared in the same way as the test bowls without introducing $M U$ contaminated Tetramin Baby Fish Food (Charterhouse Aquatics, London, UK). The mixture (eggs-food-MU) was kept in the insectary at $27^{\circ} \mathrm{C}, 75 \% \mathrm{RH}$, and $12: 12 \mathrm{LD}$ for eggs hatching. The first instars larvae progeny (L1) obtained was kept in the contaminated breeding water for ingestion of the bacteria $(M U)$ for 24 hours after which the breeding water was completely replaced with a new $M U$ free breeding water (water + food only). The L1 larvae were fed with Tetramin and bred till obtaining the second, third, and fourth instars larvae, as well as the pupae and adult mosquitoes. To avoid crosscontaminations during the experiments, all materials and consumables such as rearing bowls, rearing water, and larvae food used for mosquitoes breeding were replaced on daily basis. Rearing waters as well as Tetramin Baby Fish Food were initially tested (qPCR analysis) and confirmed free of $M U$ prior to be used in the experiments. Breeding bowls remained covered throughout larval rearing. The entire experiment was repeated thrice to ascertain the accuracy of the data.

(2) Monitoring of Infected Mosquitoes. Pools of 10 individuals per developmental stage (egg, L1, L2, L3, L4, pupae, and adult) were prepared from test and control bowls. These pools of individuals were kept in labeled Eppendorf tubes with $70 \%$ ethanol and stored at $-20^{\circ} \mathrm{C}$ for molecular screening of $M U$. In addition, we also harvested from breeding water 
TABLE 1: Distribution of field-caught mosquito species in study sites.

\begin{tabular}{|c|c|c|c|c|c|c|}
\hline \multirow{2}{*}{ Mosquito species } & \multirow{2}{*}{ Developmental stages } & \multicolumn{4}{|c|}{ Study areas } & \multirow{2}{*}{ Total } \\
\hline & & Agbahounsou & Agongbo & Agodenou & Tanongou & \\
\hline \multirow{2}{*}{ Anopheles gambiae s.l. } & Adult & 134 & 162 & 119 & 800 & 1215 \\
\hline & Larvae & 210 & 110 & 303 & 630 & 1253 \\
\hline Mansonia africana & Adult & 190 & 870 & 200 & 140 & 1400 \\
\hline \multirow{2}{*}{ Culex quinquefasciatus } & Adult & 320 & 690 & 232 & 90 & 1332 \\
\hline & Larvae & 450 & 550 & 354 & 310 & 1664 \\
\hline Aedes aegypti & Adult & 20 & 46 & 25 & 5 & 96 \\
\hline Unknown sp. & Pupae & 80 & 65 & 113 & 0 & 258 \\
\hline Total & & 1404 & 2493 & 1346 & 1975 & 7218 \\
\hline
\end{tabular}

the cuticles from the different larval molting phases and preserved them for similar molecular analysis. Finally, the third group of stored samples was constituted of small volumes $(1 \mathrm{ml})$ of breeding water collected during the entire larval developmental stages. Collected breeding waters were spun at 14,000 rpm for 5 minutes; then, the condensate was vortexed vigorously and $250 \mu \mathrm{l}$ was used for DNA extraction. The rationale of preserving cuticles and breeding water is to be certain after analysis that the bacterium was effectively ingested by the larvae and is inside the larvae system and not on its skin (due to cuticle colonization). For example, the presence of the bacterium DNA in larvae and its total absence in the water and the cuticle at a given developmental stage will imply that the bacterium was not on the larva skin (colonization of the skin) but is within/inside the larvae. For this infection monitoring experiment, preserved pools of larvae/adults were screened for $2 M U$ markers (IS2404 and KR-B which is more specific to $M U$ ). A standard curve of the $\mathrm{qPCR}$ values (Cts) and the bacterial loads was plotted and this curve was used to determine the bacterial infection rate and to monitor the presence of the bacteria at all larval developmental stages and also at the mosquitoes emergence (the adult stage).

2.6. Statistical Analysis. Statistical analysis of generated data was performed using SPSS 17.0 software (SPSS Inc., Chicago IL, USA). Chi-square test was used to set the difference in proportions (mosquitoes distribution and distribution of MU targets between localities and eggs hatching rates). Nonparametric ANOVA test (Kruskal-Wallis) was used to set the difference in means (bacterial loads and "Ct" values according to mosquitoes developmental stage), whereas the Pearson logistic regression test was used to establish the correlation between $M U$ bacterial loads and the corresponding "Ct" values (Table 5). A pool of mosquitoes (adults or larvae) was defined infected with $M U$ if found positive for the three targets (IS2404, IS2606, and KR-B) for field screened samples and two targets (IS2404 and KR) for laboratory infected samples. Two standard curves were plotted from serial dilutions of $M U$ strain (Agy99) and the Ct values for IS2404 and KR-B genes. Based on these standard curves, the cycle threshold $(\mathrm{Ct})$ cut-off was set at less than 35 cycles for IS2404 and less than 37 cycles for KR-B. Threshold for statistical significance was set at $p<5 \%$.

\section{Results}

3.1. Distribution of Mosquito' Species Collected in Studied Localities. A total of 4,043 adult mosquitoes were collected during surveyed periods in the three targeted BU endemic villages (Agongbo, Agodenou, and Agbahounsou) and the single BU nonendemic village (Tanongou). 404 pools of 10 adults were generated from sampled mosquitoes which were identified to genus and to species. Pools were grouped by identified species of mosquitoes in each village. Four mosquito species were found in surveyed localities, namely, Mansonia africana (34.63\%), Culex quinquefasciatus (32.95\%), Anopheles gambiae s.l. (30.05\%), and Aedes aegypti (2.37\%) (Table 1).

In addition to sampled adult mosquitoes, 3,175 mosquitoes larvae were collected from mosquitoes breeding sites found in the endemic villages and the control site. These larvae were used to generate 317 pools of 10 larvae. Larvae identified in the endemic sites included $60.6 \%$ of Culex quinquefasciatus, $27.86 \%$ of Anopheles gambiae s.l., and $11.54 \%$ of the pupal stage of an unknown species (unknown sp.). In the nonendemic control site only two larvae of two genera were detected, Anopheles gambiae s.l. (67.02\%) and Culex quinquefasciatus (32.98\%) (Table 1).

\subsection{Screening of IS2404, IS2606, and KR-B Targets in Wild Populations of Mosquitoes from Endemic and Nonendemic Localities}

3.2.1. Screening of IS2404. Out of 301 pools of adult mosquitoes (3,010 mosquitoes) from endemic villages subjected to real time quantitative PCR analysis, 26 pools $(8.63 \%)$ were found positive to IS2404 target (Table 2). At Agbahounsou, 8 pools (12.12\%) of mosquitoes were found positive to IS2404, 12 pools (6.82\%) at Agongbo, and 6 pools (10.17\%) at Agodenou for this same molecular marker. Unexpectedly, we recorded an identical trend of positive number of pools $(10 / 103,9.7 \%)$ in samples from the nonendemic control site (Table 2).

Out of 223 pools of collected mosquitoes larvae $(2,235$ mosquitoes larvae) from endemic villages subjected to qPCR analysis, 39 pools (17.49\%) were found positive to IS2404 target with 10 pools (13.51\%) at Agbahounsou, 24 pools (32.88\%) at Agongbo, and 5 pools (6.58\%) at Agodenou. At Tanongou 
TABLE 2: Distribution of $M U$ targets in field-caught adult mosquitoes.

\begin{tabular}{|c|c|c|c|c|c|c|c|c|c|}
\hline \multirow{2}{*}{\multicolumn{2}{|c|}{ Study sites }} & \multirow{2}{*}{$\begin{array}{c}\text { Pools of } 10 \text { adult } \\
\text { mosquitoes analyzed }\end{array}$} & \multicolumn{2}{|c|}{ IS2404-qPCR } & \multicolumn{2}{|c|}{$K R$-qPCR } & \multicolumn{2}{|c|}{ IS2606-qPCR } & \multirow{2}{*}{ MU distribution } \\
\hline & & & Positive & $P(\%)$ & Positive & $P(\%)$ & Positive & $P(\%)$ & \\
\hline \multirow{3}{*}{ BU endemic villages } & Agbahounsou & 66 & 8 & 12.12 & 1 & 12.5 & 0 & - & Absent \\
\hline & Agongbo & 176 & 12 & 6.82 & 0 & - & 0 & - & Absent \\
\hline & Agodenou & 59 & 6 & 10.17 & 0 & - & 0 & - & Absent \\
\hline Total & & 301 & 26 & 8.63 & 1 & 12.5 & 0 & - & Absent \\
\hline BU nonendemic village & Tanongou & 103 & 10 & 9.7 & 0 & - & 0 & - & Absent \\
\hline
\end{tabular}

P: percentage of targets distribution. No statistical difference was found in the distribution of IS2404 target between the endemic and nonendemic localities $(p=0.601)$.

TABLE 3: Distribution of $M U$ targets in field collected mosquitoes larvae.

\begin{tabular}{|c|c|c|c|c|c|c|c|c|c|}
\hline \multirow{2}{*}{\multicolumn{2}{|c|}{ Study sites }} & \multirow{2}{*}{$\begin{array}{l}\text { Pools of } 10 \text { mosquito } \\
\text { larvae analyzed }\end{array}$} & \multicolumn{2}{|c|}{ IS2404-qPCR } & \multicolumn{2}{|c|}{$K R-\mathrm{qPCR}$} & \multicolumn{2}{|c|}{ IS2606-qPCR } & \multirow{2}{*}{ MU distribution } \\
\hline & & & Positive & $P(\%)$ & Positive & $P(\%)$ & Positive & $P(\%)$ & \\
\hline \multirow{3}{*}{ BU endemic villages } & Agbahounsou & 74 & 10 & 13.51 & 0 & - & 2 & 16.67 & Absent \\
\hline & Agongbo & 73 & 24 & 32.88 & 0 & - & 1 & 5.26 & Absent \\
\hline & Agodenou & 76 & 5 & 6.58 & 0 & - & 0 & - & Absent \\
\hline Total & & 223 & 39 & 17.49 & 0 & - & 0 & - & Absent \\
\hline BU nonendemic village & Tanongou & 94 & 11 & 11.70 & 0 & - & 0 & - & Absent \\
\hline
\end{tabular}

P: percentage of targets distribution. No statistical difference was found in the distribution of IS2404 target between the endemic and nonendemic localities $(p=0.347)$.

the control site, 11 pools (11.70\%) out of 94 tested from 940 mosquitoes larvae were found positive to IS2404 target (Table 3). No statistical difference was found in the distribution of this target between the test and control localities in both adult and larval mosquitoes $(p>0.05)$.

3.2.2. Screening of IS2606. Out of 26 pools of adult mosquitoes tested positive to IS2404 target in the three endemic villages (Agongbo, Agodenou, and Agbahounsou), none was found to be positive for the IS2606 target. The same finding was observed after real time quantitative PCR analysis of the 10 pools of mosquitoes tested positive to IS2404 in the control site. No sample was found positive to IS2606 in the nonendemic site (Table 2).

However, the IS2606 target was detected in 3/39 (7.7\%) pools of larvae which were positive to IS2404 target in the endemic sites (Table 3). None of the IS2404 positive mosquitoes larvae (positive pools) from one endemic site (Agodenou) or the control site were positive for the IS2606 target (Table 3).

3.2.3. Screening of KR-B. Only one pool (3.84\%) out of 26 pools of adult mosquitoes tested positive to IS2404 target was found positive for the $K R-B$ target in samples from the endemic villages. This single KR-B positive pool of mosquitoes belonged to the genus Anopheles caught at Agbahounsou. However, it is worth indicating that this unique KR-B ( $M U$ plasmid marker) positive pool was not found positive to the IS2606. None of the 10 pools of IS2404 positive mosquitoes from the control site tested positive for the $K R-B$ target (Table 2).
In addition, none of the mosquitoes larvae that tested positive to IS2404 target was found to be positive for the $K R-B$ in both the endemic and the nonendemic areas (Table 3).

3.2.4. Summary of Results from the Screening of the 3 Targets Related to the Presence of MU in Analyzed Wild Populations of Mosquitoes. None of the adult and larvae pools was found to contain the three $M U$ targets (IS2404, IS2606, and $K R-B)$. This demonstrated the absence of MU in the wild mosquitoes populations in the endemic region surveyed. Although the IS2404 target was detected in mosquitoes caught in the nonendemic village, these samples also lacked the three targets related to the presence of $M U$ and most likely represent the presence of other environmental mycobacterial species (Tables 2 and 3 ).

3.3. Analysis of the Low Capability of Mosquitoes to Pick and Host MU from Larval to Adult Stages. Following the inoculation of Anopheles kisumu eggs in simulated laboratory breeding experiment (bowls containing water, larvae food) fed with $M U$, we recorded an average hatching rate of 94.010 $\pm 1.289 \%$ in the 4 bowls which served as "test bowls" (water + food + MU + eggs of An. kisumu) and an average hatching rate of $93.87 \pm 0.546 \%$ in the 2 bowls serving as "control bowls" (water + food + eggs of An. kisumu). Overall, the bacterial load decreased throughout the experiment from the young (1st instars larvae) to the old (pupae and adult stages) developmental stages of An. kisumu (Figure 2). No significance difference was observed in the decrease of the bacterial loads throughout the mosquitoes developmental stages in mosquitoes samples $(p=0.220)$, cuticles $(p=$ $0.199)$, and breeding waters $(p=0.092)$. 


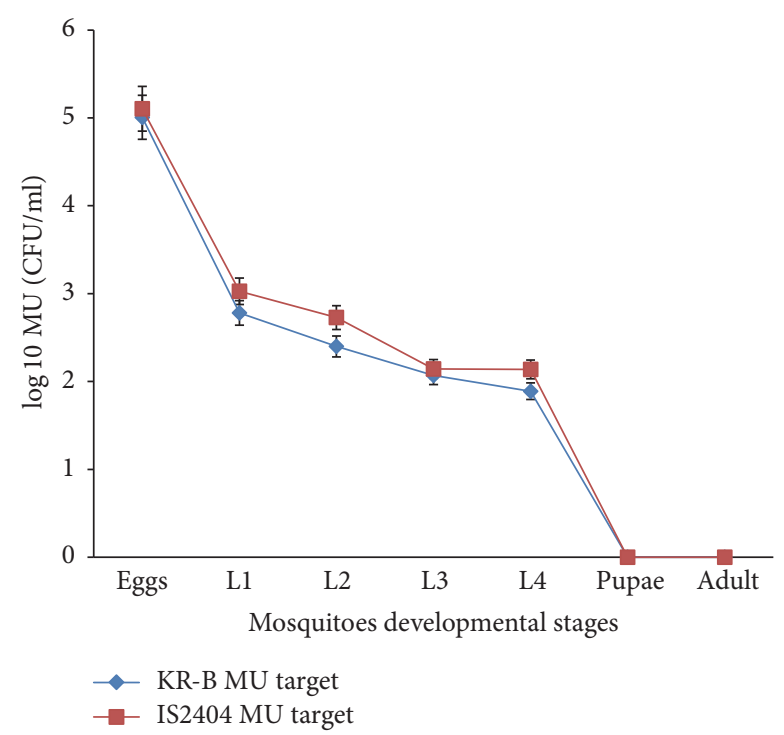

FIGURE 2: Distribution of average bacterial load during mosquito developmental stages. L1, L2, L3, and L4 correspond to first, second, third, and fourth instars larvae, respectively. Values are given with error bars at $5 \%$.

3.3.1. Distribution of $M U$ in First Instars Larvae (L1) of Anopheles kisumu. Randomly selected L1 larvae from the 4 "test" bowls ( 1 pool of $10 \mathrm{~L} 1$ larvae per bowl, making a total of 4 pools for the 4 bowls) showed after qPCR analysis that all 4 pools of L1 mosquitoes larvae were infected/colonized by $M U$. Real time PCR analysis targeting the KR-B domain of $M U$ revealed a mean $\mathrm{Ct}$ value of $31.592 \pm 3.151$ cycles which corresponds to a mean bacterial load of $E+2.779 \pm E+$ $0.817 \mathrm{CFU} / \mathrm{ml}$ in $\mathrm{L} 1$ larvae. The analysis of cuticles (1 pool of 10 cuticles from each bowl, making a total of 4 pools of cuticles from test bowls) released from the metamorphosis of L1 larvae revealed the presence of $M U$ in all the 4 pools from "test" bowls (100\% infection rate with $M U, 4 / 4$ pools). The mean Ct value of $36.516 \pm 2.096$ cycles corresponding to the mean bacterial load of $E+1.503 \pm E+0.523 \mathrm{CFU} / \mathrm{ml}$ was from L1 released cuticles. When the breeding water was analyzed during L1 larval development, the mean planktonic bacterial load in the water was $E+3.034 \pm E+1.024 \mathrm{CFU} / \mathrm{ml}$, corresponding to a mean $\mathrm{Ct}$ value of $30.610 \pm 2.801$ cycles. As observed with larvae and cuticles, $M U$ was also detected in all breeding waters (4/4) during the L1 developmental stage of An. kisumu. MU was not found in L1 larvae, cuticles, or breeding water collected from the 2 bowls constituting the "control group" (Table 4).

3.3.2. Distribution of $M U$ in Second Instars Larvae (L2) of Anopheles kisumu. Randomly selected L2 larvae pools from the "test" bowls and controls were collected as was the case for the L1 larval stages. All 4 test pools of L2 mosquitoes larvae were infected or colonized by $M U$. Real time PCR of the $M U$ KR-B domain of $M U$ yielded a mean Ct value of $33.063 \pm$ 2.984 cycles equivalents to a mean bacterial load of $E+2.399 \pm$ $E+0.773 \mathrm{CFU} / \mathrm{ml}$. Cuticles released from the metamorphosis of L2 larvae had $M U$ in 3/4 (75\% infection/colonization rate) pools from "test" bowls. The mean Ct value of $36.823 \pm 1.652$ cycles equivalent to a mean bacterial load of $E+1.424 \pm E+$ $0.428 \mathrm{CFU} / \mathrm{ml}$ was recorded. When the breeding water was analyzed during L2 larval development, the estimated mean planktonic bacterial load found in the water was $E+2.705 \pm$ $E+0.680 \mathrm{CFU} / \mathrm{ml}$. Bacteria was found in all 4 tests during the L2 developmental stage of An. kisumu. Traces of bacteria were not found in L2 larvae, cuticles, or breeding water in the "control group" (Table 4).

3.3.3. Distribution of $M U$ in Third Instars Larvae (L3) of Anopheles kisumu. Randomly selected L3 larvae pools as previously described for L1 and L2 showed that all 4 pools of L3 mosquitoes larvae were infected or colonized by $M U$ and had mean Ct values to the KR-B region of $34.33 \pm 3.349$ cycles equivalent to a mean bacterial load of $E+2.070 \pm$ $E+0.031 \mathrm{CFU} / \mathrm{ml}$ in L3. The analysis of cuticles only showed the presence of $M U$ in 1/4 (25\%) pools from "test" bowls. The breeding water during L3 larval development had an estimated mean planktonic bacterial load of $E+2.277 \pm E+$ $0.023 \mathrm{CFU} / \mathrm{ml}$. MU was not detected in any of the control group samples (Table 4 ).

3.3.4. Distribution of $M U$ in Fourth Instars Larvae (L4) of Anopheles kisumu. Randomly selected L4 larvae from the 4 "test" bowls showed that only 3/4 (75\%) pools of L4 mosquitoes larvae were infected or colonized by $M U$. The mean $\mathrm{Ct}$ value of $35.03 \pm 1.177$ cycles equivalent to a mean bacterial load of $E+1.88 \pm E+0.441 \mathrm{CFU} / \mathrm{ml}$ was recorded in L4. $M U$ was not detected in samples of cuticles released from the metamorphosis of L4 larvae. Breeding water samples had estimated mean planktonic bacterial loads of $E+1.652 \pm$ $E+0.019 \mathrm{CFU} / \mathrm{ml}$. Three out of $4(75 \%)$ breeding water samples were contaminated with the bacteria during the L4 developmental stage. $M U$ was not detected in any of the samples from the L4 control group samples (Table 4).

3.3.5. Distribution of MU in Pupae Stages of Anopheles kisumu. $M U$ was not detected from the randomly selected pupae from the 4 "test" bowls. In addition, $M U$ was not detected in the cuticles released from the emergence of adult mosquitoes from pupae. Only one out of 4 (25\%) breeding water samples was contaminated with the bacteria $M U$ during pupae developmental stage, with a KR-B Ct value of 35.47 cycles. As above, $M U$ was not detected in samples constituting the control group (Table 4).

3.3.6. Distribution of MU in Adult Stages of Anopheles kisumu. Overall, $M U$ was not detected in any of adult stage samples or their controls (Table 4).

\section{Discussion}

4.1. Wild Populations of Mosquitoes Are Unlikely to Be $M U$ Reservoirs in Sedje-Denou. According to WHO, a reservoir is any person, animal, arthropod, plant, soil, or substance, or a combination of these, in which an infectious agent lives and multiplies and where it reproduces itself in such a manner that it can be transmitted to a susceptible host [38]. Difficulties to cultivate Mycobacterium ulcerans (MU) from 


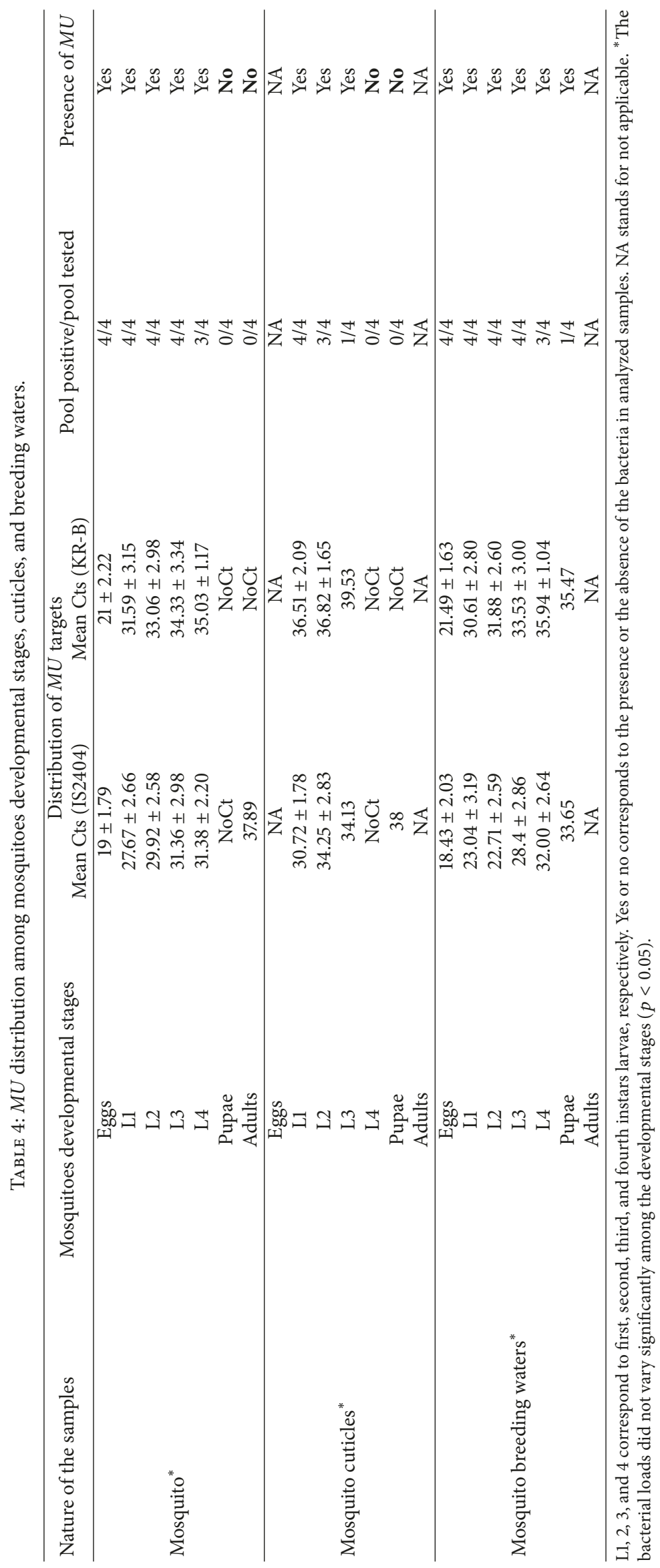


TABLE 5: Characteristics of the standard curves linking "Ct" values of $M U$ targets and corresponding bacterial loads ( $M U$ Agy99 serial dilutions).

\begin{tabular}{lccc}
\hline$M U$ targets & Regression coefficient $\left(R^{2}\right)$ & Regression equation $(95 \% \mathrm{CI})$ & $p$ value \\
\hline IS2404 & 0.9955 & $Y=9.6569-0.2396 X$ & 0.000008 \\
KR-B & 0.9968 & $Y=10.9682-0.2592 X$ & 0.000004 \\
\hline
\end{tabular}

Independent variable, Ct values (cycle threshold); dependent variable, log10 $M U(\mathrm{CFU} / \mathrm{ml})$.

contaminated environmental samples remains the main challenge in the identification of reproductive reservoir(s) for this Mycobacterium as well as the understanding of its transmission mode(s) from the $M U$ contaminated environment to humans. Most environmental samples that have been identified with $M U$ have been classified as "potential reservoirs" $[1,39]$. Aquatic water bugs have been shown to replicate $M U$ in their salivary glands [40] and $M U$ has been successfully recovered by culture from theses insects [11], and thus, the "reservoir" capacity of other "suspected organisms" remains unclear. The aquatic environment has been identified as the most predominant source of $M U$ contamination [12-18, 31, 41-45]. This research was conducted in the wet agroecosystem of Sedje-Denou region and more specifically in three endemic villages which served as test sites for this study. From the three thousand and ten adult mosquitoes subjected to real time PCR, twenty-six pools (8.64\%) were positive to the insertion sequence IS2404, which is not specific enough to infer the presence of $M U$. We recorded the presence of this insertion (IS2404) in mosquitoes samples collected from nonendemic location (Tanongou in the Northern Benin). These results further highlight the nonspecificity of this marker for $M U$ detection from environmental samples [4, $36,45]$. The use of two additional targets (IS2606 and KR) to increase the specificity of $M U$ detection in our study showed that none of the mosquitoes tested to be simultaneously positive for all three targets. These results certainly confirm the low capability of wild mosquitoes populations to carry $M U$ as previously described by others in this same Southern region of Benin [25]. However, our data seems to contradict works conducted in Australia which detected $M U$ in mosquitoes samples [7, 19-23, 26]. Johnson et al. [22] described the contamination of mosquito species by $M U$ as a consequence of resting and feeding or breeding in storm water drains, whereas Wallace et al. [26], in an experimental study, suggested an unlikely role for mosquitoes as BU biological vectors. In their study using mice and both natural and anthropogenic forms of inoculation, they emphasized that reducing exposure to insect bites and destroying mosquitoes breeding sites around households would break the chain of BU transmission [28]. These series of studies on the role of mosquitoes in the transmission of $M U$ show the need of further investigations whether mosquitoes can act as both reservoir and vector of $M U$. In this current study, none of the 2,235 mosquitoes larvae collected from both endemic and nonendemic areas for $\mathrm{BU}$ were found to be positive for $M U$, suggesting that mosquitoes larvae in the wild were unlikely to be reservoirs for $M U$. Although our results generated from wild mosquitoes populations are in favor of previous studies conducted in Benin which revealed the inability of mosquitoes to be involved in $M U$ transmission [25], a laboratory designed experimental model was designed to better understand the poor implication of mosquitoes in increased number of BU cases in West and Central Africa $[1,6]$.

4.2. Inability of An. kisumu Larvae to Pick Up MU from Their Environment and Remain Colonized through the Larval Developmental Stages to the Adult Stage. Mosquitoes (Culicidae) development, as characteristic of all holometabolous insects, proceeds through embryonic, larval, pupal, and adult stages that reflect considerable morphological and physiological differences [34]. These stages exhibit distinct niches; larvae and pupae are aquatic while adults are free-flying and terrestrial. In mosquitoes vectors, vertical transmission has been demonstrated for certain pathogens which include yellow fever virus, dengue virus, St. Louis encephalitis virus, Japanese encephalitis virus, and West Nile virus (WNV) [46]. Vertical transmission involves the transmission of pathogens from female mosquitoes to their offspring. The laboratory experimental model showed that mosquitoes larvae readily ingested $M U$ and that $M U$ colonized the larval stages through the pupal stage. However, at the pupae series of high energy demanding [47], metabolism taking place in the mosquitoes certainly affects $M U$ development leading to the clearing of $M U$ colonization by the end of pupation and at the adult stage (Figure 2). Our research demonstrated the total absence of $M U$ at both pupae and adult stages as reported by Wallace et al. [28] and, thus, highlights the inability of these biting dipterans to act as a good vector/host of $M U$ in an endemic environment. Results from this laboratory based experiment are consistent with those obtained from the analysis of thousands of wild populations of mosquitoes collected in the endemic locations which did not show any $M U$ colonization through molecular testing. Data published by Wallace et al. [26] suggested that $M U$ is unlikely to persist in the mosquito's body system, a behavior which stands as a natural protective mechanism of mosquitoes to bacterial infections. According to Hoxmeier et al. [48], the contamination of Anopheles gambiae mosquitoes with $M U$ resulted in disruptions to phospholipid metabolic pathways in the mosquitoes, especially the use of glycolipid molecules. Moreover, glycolipids are actively involved in signaling and are mediators in cellular and immune processes [49]. The disruption of synthesis of this molecule probably has a negative impact on the various interactions between $M U$ cells and Anopheles and the poor capability of mosquitoes to serve as biological vectors for $M U$ [45]. Instead of acting as biological vectors for $M U$ as described in this study, mosquitoes might act as mechanical vectors as recently described in an experimental study with Aedes notoscriptus and BALB/C mice [28]. However, 
mechanical transmission of $M U$ seems to happen only after skin trauma either by an insect bite or by any other environmental stress (e.g., a thorn, penetrating wood splinters, and scorpion stings) [29]. The traumatized skin should initially be colonized by $M U$, a phenomenon that could naturally happen during repetitive contacts with the risk environments such as water bodies or contaminated biofilms $[1,17,28]$. Furthermore, in behavioral study with Aedes aegypti, Sanders et al. [50] suggested that if a biofilm of $M U$ was on a person, the bacteria may be attracting mosquitoes which in return would lead to a puncture insertion of $M U$ as recently reported by Wallace et al. [28]. Although mechanical transmission of $M U$ stands as a common mechanism that could correlate transmission studies from both Africa and Australia, Williamson et al. [30] recently established that abrasions (trauma) of the skin in Guinea pig models and subsequent application of $M U$ are not sufficient enough to cause an ulcer. Further laboratory and epidemiological studies are therefore required to understand the extent of the mechanical transmission of $M U$ and how frequent animals including humans can carry and remain colonized with $M U$ on their skin to facilitate such transmission mode. $M U$ could be traced from the risk environments to humans or animals directly after they had contact with colonized environments. In such hypothetical situations and for preventive measures, individuals from endemic areas should remain aware and avoid frequent contacts with mosquito's bites by sleeping under mosquitoes bed nets, wearing protective clothing while farming or using clean water for bathing and cleaning $[1,7,15,17,19,28]$.

Mosquitoes larvae breeding in an $M U$ contaminated water body are capable of ingesting this bacterium as shown by Hoxmeier et al. [48] and Wallace et al. [26] in Aedes aegypti, Aedes albopictus, Culex restuans, and Ochlerotatus triseriatus larvae. Although several experimental studies have established the potential of predaceous aquatic insects to temporally maintain $M U$ during their developmental stages in water $[37,40]$, our findings in addition to confirming these previous results also show that $M U$ colonization of mosquitoes larvae is very temporal as larvae system is capable of clearing the bacterial load during pupae and adult developmental stages. The vertical transmission of $M U$ therefore seems not to be effective in mosquitoes populations as documented with several viruses. The noncontamination/colonization of field-caught mosquito species by $M U$ as found in this study might suggest that mosquitoes are unable to move $M U$ from one source to another in endemic areas in Benin.

\section{Conclusion}

This study revealed the absence of $M U$ in hematophagous mosquitoes trapped in households in BU endemic locations in the Sedje-Denou division in Benin. Using an experimental model, we also showed the inability of laboratory infected or colonized An. kisumu larvae to transfer the bacteria to their pupae and the emerging adults. This low ability of mosquitoes to vertically transmit $M U$ pathogens to their offspring coupled with the absence of $M U$ in field-caught mosquitoes further highlights the low probability of these biting insects as biological vectors for $M U$ in endemic villages in Benin. Mosquitoes may therefore not be involved in the dissemination of this pathogen from the risk environments to humans in investigated areas. However, further studies should be performed to evaluate their mechanical implication, before completely excluding whether they are involved or not in the transmission cycle of this emerging disease.

\section{Conflicts of Interest}

The authors declare that they have no conflicts of interest.

\section{Authors' Contributions}

Rousseau Djouaka, Francis Zeukeng, Jude Daiga Bigoga, Wilfred Fon Mbacham, Solange E. Kakou-Ngazoa, and Anthony Ablordey conceived and designed the experiments. Rousseau Djouaka, Francis Zeukeng, Genevieve Tchigossou, Sylla Aboubacar, Romaric Akoton, David N'golo Coulibaly, Sodjinin Jean-Eudes Tchebe, Clavella Nantcho Nguepdjo, Romaric Akoton, Innocent Djegbe, and Solange E. KakouNgazoa participated in collection and analyses of the data. Rousseau Djouaka, Francis Zeukeng, Razack Adeoti, Sodjinin Jean-Eudes Tchebe, and Innocent Djegbe performed the experimental study on the vertical transmission of $M$. ulcerans in mosquitoes. Jude Daiga Bigoga, Wilfred Fon Mbacham, Solange E. Kakou-Ngazoa, Manuele Tamo, and Anthony Ablordey supervised the experiments. Rousseau Djouaka and Francis Zeukeng performed the statistical analysis of the data. Rousseau Djouaka and Francis Zeukeng drafted the manuscript. Anthony Ablordey, Solange E. Kakou-Ngazoa, and Manuele Tamo made critical revisions of the manuscript. All authors read and approved the final manuscript.

\section{Acknowledgments}

This work was supported in part by a research grant from the International Society for Infectious Diseases (ISID, United States) attributed to Francis Zeukeng and the AgroEcoHealth Platform of the International Institute of Tropical Agriculture (IITA-Benin). The authors express their gratitude to household heads and community health workers of the Sedje-Denou and Tanongou localities for their assistance during sample collections. They also extend their gratitude to Professor Dorothy Yeboah-Manu and Dr. Anthony Ablordey for providing the bacterial strain used in this study, their assistance, and the supervision of Francis Zeukeng internships in the Bacteriology Department of Noguchi Memorial Institute for Medical Research (NMIMR) in Accra, Ghana.

\section{References}

[1] R. W. Merritt, E. D. Walker, P. L. C. Small et al., "Ecology and transmission of Buruli ulcer disease: a systematic review," PLoS Neglected Tropical Diseases, vol. 4, no. 12, p. e911, 2010.

[2] G. E. Sopoh, R. C. Johnson, A. Chauty et al., "Buruli ulcer surveillance, Benin, 2003-2005," Emerging Infectious Diseases, vol. 13, no. 9, pp. 1374-1376, 2007.

[3] M. Debacker, J. Aguiar, C. Steunou et al., "Mycobacterium ulcerans disease (Buruli ulcer) in rural hospital, southern Benin, 
1997-2001," Emerging Infectious Diseases, vol. 10, no. 8, pp. 13911398, 2004.

[4] World Health Organization, Laboratory Diagnosis of Buruli Ulcer: A Manual for Health Care Providers, WHO, Geneva, Switzerland, 2014.

[5] K. M. George, D. Chatterjee, G. Gunawardana et al., "Mycolactone: a polyketide toxin from Mycobacterium ulcerans required for virulence," Science, vol. 283, no. 5403, pp. 854-857, 1999.

[6] World Health Organization, Buruli ulcer: Number of New Cases of Buruli Ulcer Reported (Per Year), WHO, Geneva, Switzerland, 2011, http://apps.who.int/neglected_diseases/ntddata/buruli/buruli.html.

[7] T. Y. J. Quek, E. Athan, M. J. Henry et al., "Risk factors for Mycobacterium ulcerans infection, southeastern Australia," Emerging Infectious Diseases, vol. 13, no. 11, pp. 1661-1666, 2007.

[8] World Health Organization, Buruli ulcer (Mycobacterium ulcerans infection), WHO Media centre, 2015, http://www.who .int/mediacentre/factsheets/fs199/en/.

[9] H. R. Williamson, M. E. Benbow, L. P. Campbell et al., "Detection of Mycobacterium ulcerans in the environment predicts prevalence of Buruli ulcer in Benin," PLoS Neglected Tropical Diseases, vol. 6, no. 1, Article ID e1506, 2012.

[10] G. Francis, M. Whitby, and M. Woods, "Mycobacterium ulcerans infection: a rediscovered focus in the Capricorn Coast region of central Queensland," Medical Journal of Australia, vol. 185, no. 3, pp. 179-180, 2006.

[11] F. Portaels, W. M. Meyers, A. Ablordey et al., "First cultivation and characterization of Mycobacterium ulcerans from the environment," PLoS Neglected Tropical Diseases, vol. 2, no. 3, article no. el78, 2008.

[12] M. W. Bratschi, M. T. Ruf, A. Andreoli et al., "Mycobacterium ulcerans persistence at a village water source of Buruli ulcer patients," PLoS Neglected Tropical Diseases, vol. 8, no. 3, Article ID e2756, 2014.

[13] J. Landier, J. Gaudart, K. Carolan et al., "Spatio-temporal patterns and landscape-associated risk of buruli ulcer in Akonolinga, Cameroon," PLoS Neglected Tropical Diseases, vol. 8, no. 9, p. e3123, 2014.

[14] G. E. Sopoh, Y. T. Barogui, R. C. Johnson et al., "Family relationship, water contact and occurrence of buruli ulcer in Benin," PLoS Neglected Tropical Diseases, vol. 4, no. 7, article e746, 2010.

[15] M. Debacker, F. Portaels, J. Aguiar et al., "Risk factors for buruli ulcer, Benin,” Emerging Infectious Diseases, vol. 12, no. 9, pp. 1325-1331, 2006.

[16] R. W. Merritt, M. E. Benbow, and P. L. C. Small, "Unraveling an emerging disease associated with disturbed aquatic environments: the case of Buruli ulcer," Frontiers in Ecology and the Environment, vol. 3, no. 6, pp. 323-331, 2005.

[17] H. Aiga, T. Amano, S. Cairncross, J. A. Domako, O.-K. Nanas, and S. Coleman, "Assessing water-related risk factors for Buruli ulcer: a case-control study in Ghana," American Journal of Tropical Medicine and Hygiene, vol. 71, no. 4, pp. 387-392, 2004.

[18] L. Marsollier, J. Aubry, J.-P. Saint-André et al., "Ecology and transmission of Mycobacterium ulcerans," Pathologie Biologie, vol. 51, no. 8-9, pp. 490-495, 2003.

[19] C. J. Lavender, J. A. M. Fyfe, J. Azuolas et al., "Risk of Buruli ulcer and detection of Mycobacterium ulcerans in mosquitoes in Southeastern Australia," PLoS Neglected Tropical Diseases, vol. 5, no. 9, Article ID el305, 2011.

[20] P. D. R. Johnson and C. J. Lavender, "Correlation between buruli Ulcer and vector-borne notifiable diseases, Victoria, Australia," Emerging Infectious Diseases, vol. 15, no. 4, pp. 614-615, 2009.
[21] T. Y. J. Quek, J. M. Henry, A. J. Pasco et al., "Mycobacterium ulcerans infection: factors influencing diagnostic delay," Medical Journal of Australia, vol. 187, pp. 561-563, 2007.

[22] P. D. R. Johnson, J. Azuolas, C. J. Lavender et al., "Mycobacterium ulcerans in mosquitoes captured during outbreak of Buruli ulcer, southeastern Australia," Emerging Infectious Diseases, vol. 13, no. 11, pp. 1653-1660, 2007.

[23] P. D. R. Johnson, T. Stinear, and P. L. C. Small, "Buruli ulcer ( $M$. ulcerans infection): new insights, new hope for disease control," PLoS Medicine, vol. 2, no. 4, article e108, 2005.

[24] P. Le Gall, J. Landier, J. De Matha Ndengue et al., Detection of Mycobacterium ulcerans in domestic arthropods in a Buruli ulcer endemic site, Akonolinga, Cameroon, World Health Organization, Geneva, Switzerland, 2015.

[25] B. Zogo, A. Djenontin, K. Carolan et al., "A field study in Benin to investigate the role of mosquitoes and other flying insects in the ecology of mycobacterium ulcerans," PLoS Neglected Tropical Diseases, vol. 9, no. 7, Article ID e0003941, 2015.

[26] J. R. Wallace, M. C. Gordon, L. Hartsell et al., "Interaction of Mycobacterium ulcerans with mosquito species: implications for transmission and trophic relationships," Applied and Environmental Microbiology, vol. 76, no. 18, pp. 6215-6222, 2010.

[27] B. F. Eldridge, "Mosquitoes, the culicidae," in Biology of disease vectors, W. C. Marquardt, Ed., p. 108, Elsevier Academic Press, New York, NY, USA, 2005.

[28] J. R. Wallace, K. M. Mangas, J. L. Porter et al., "Mycobacterium ulcerans low infectious dose and mechanical transmission support insect bites and puncturing injuries in the spread of Buruli ulcer," PLOS Neglected Tropical Diseases, vol. 11, no. 4, p. e0005553, 2017.

[29] W. M. Meyers, W. M. Shelly, D. H. Connor, and E. K. Meyers, "Human Mycobacterium ulcerans infections developing at sites of trauma to skin," American Journal of Tropical Medicine and Hygiene, vol. 23, no. 5, pp. 919-923, 1974.

[30] H. R. Williamson, L. Mosi, R. Donnell, M. Aqqad, R. W. Merritt, and P. L. C. Small, "Mycobacterium ulcerans fails to infect through skin abrasions in a guinea pig infection model: implications for transmission," PLoS Neglected Tropical Diseases, vol. 8, no. 4, Article ID e2770, 2014.

[31] T. Wagner, M. E. Benbow, T. O. Brenden, J. Qi, and R. C. Johnson, "Buruli ulcer disease prevalence in Benin, West Africa: associations with land use/cover and the identification of disease clusters," International Journal of Health Geographics, vol. 7, article 25, 2008.

[32] World Health Organization, Guidelines for Testing Mosquito Adulticides for Indoor Residual Spraying and Treatment of Mosquito Nets, WHO, Geneva, Switzerland, 2006.

[33] F. Edwards, "Mosquitoes of the Ethiopian Region III," in Culicine adults and pupae, B. M. N. Hist, Ed., London, UK, 1941.

[34] World Health Organization, Entomologie Du Paludisme Et Contrôle Des Vecteurs, Guide Du Stagiaire, vol. 1, part 1, WHO, Geneva, Switzerland, 2003.

[35] J. Sambrook and D. W. Russell, "Purification of nucleic acids by extraction with phenol: chloroform," in Commonly Used Techniques in Molecular Cloning, J. Sambrook and D. W. Russell, Eds., vol. 3, appendix 8, Cold Spring Harbor Laboratory Press, Cold Spring Harbor, NY, USA, 3rd edition, 2001.

[36] J. A. M. Fyfe, C. J. Lavender, P. D. R. Johnson et al., "Development and application of two multiplex real-time PCR assays for the detection of Mycobacterium ulcerans in clinical and environmental samples," Applied and Environmental Microbiology, vol. 73, no. 15, pp. 4733-4740, 2007. 
[37] T. P. Stinear, T. Seemann, S. Pidot et al., "Reductive evolution and niche adaptation inferred from the genome of Mycobacterium ulcerans, the causative agent of Buruli ulcer," Genome Research, vol. 17, no. 2, pp. 192-200, 2007.

[38] World Health Organization, Yellow Fever, WHO, Geneva, Switzerland, 1998, http://www.who.ch/gpv-documents/.

[39] S. Gryseels, D. Amissah, L. Durnez et al., "Amoebae as potential environmental hosts for Mycobacterium ulcerans and other mycobacteria, but doubtful actors in buruli ulcer epidemiology," PLoS Neglected Tropical Diseases, vol. 6, no. 8, Article ID e1764, 2012.

[40] L. Marsollier, R. Robert, J. Aubry et al., "Aquatic insects as a vector for Mycobacterium ulcerans," Applied and Environmental Microbiology, vol. 68, no. 9, pp. 4623-4628, 2002.

[41] J. Hayman, "Postulated epidemiology of Mycobacterium ulcerans infection," International Journal of Epidemiology, vol. 20, no. 4, pp. 1093-1098, 1991.

[42] K. Kibadi, M. Panda, J.-J. M. Tamfum et al., "New foci of buruli ulcer, Angola and Democratic Republic of Congo," Emerging Infectious Diseases, vol. 14, no. 11, pp. 1790-1792, 2008.

[43] A. A. Duker, F. Portaels, and M. Hale, "Pathways of Mycobacterium ulcerans infection: a review," Environment International, vol. 32, no. 4, pp. 567-573, 2006.

[44] F. Portaels, K. Chemlal, P. Elsen et al., "Mycobacterium ulcerans in wild animals," Revue Scientifique et Technique de l'OIE, vol. 20, no. 1, pp. 252-264, 2001.

[45] A. L. Morris, J.-F. Guegan, D. Andreou et al., "Deforestationdriven food-web collapse linked to emerging tropical infectious disease, Mycobacterium ulcerans," Science Advances, vol. 2, no. 12, p. e1600387, 2016.

[46] I. Unlu, A. J. MacKay, A. Roy, M. M. Yates, and L. D. Foil, "Evidence of vertical transmission of West Nile virus in field-collected mosquitoes," Journal of Vector Ecology, vol. 35, no. 1, pp. 95-99, 2010.

[47] B. W. Harker, S. K. Behura, B. S. Debruyn et al., "Stage-specific transcription during development of Aedes aegypti," BMC Developmental Biology, vol. 13, no. 1, article 29, 2013.

[48] J. C. Hoxmeier, B. D. Thompson, C. D. Broeckling et al., "Analysis of the metabolome of Anopheles gambiae mosquito after exposure to Mycobacterium ulcerans," Scientific Reports, vol. 5, article 9242, 2015.

[49] G. C. Atella and M. Shahabuddin, "Differential partitioning of maternal fatty acid and phospholipid in neonate mosquito larvae," Journal of Experimental Biology, vol. 205, no. 23, pp. 3623-3630, 2002.

[50] M. L. Sanders, H. R. Jordan, C. Serewis-Pond et al., “Mycobacterium ulcerans toxin, mycolactone may enhance host-seeking and oviposition behaviour by Aedes aegypti (L.) (Diptera: Culicidae)," Environmental Microbiology, vol. 19, no. 5, pp. 17501760, 2017. 


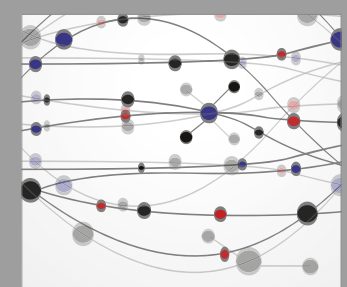

The Scientific World Journal
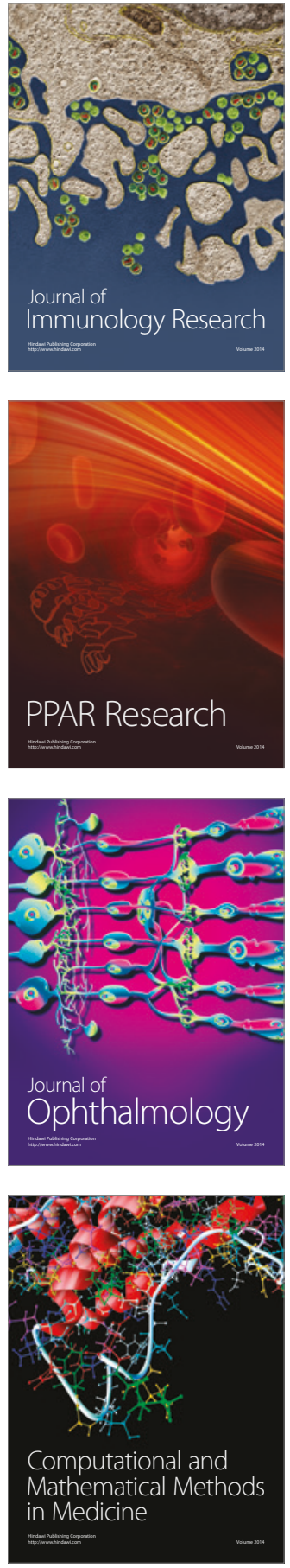

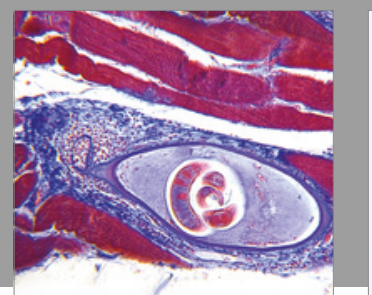

Gastroenterology Research and Practice
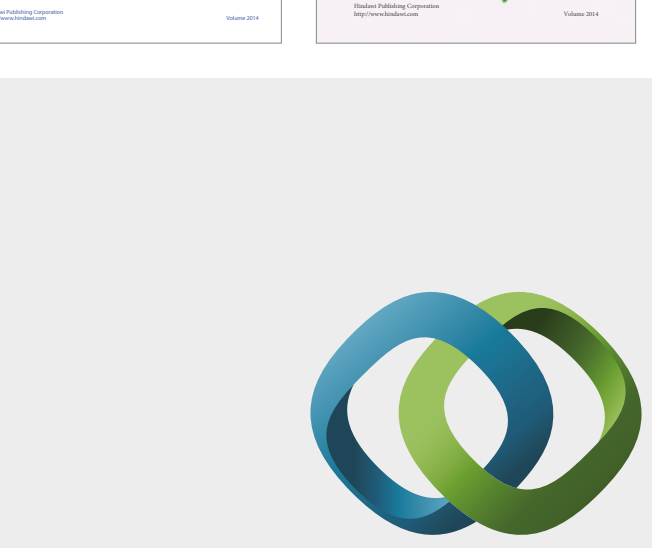

\section{Hindawi}

Submit your manuscripts at

https://www.hindawi.com
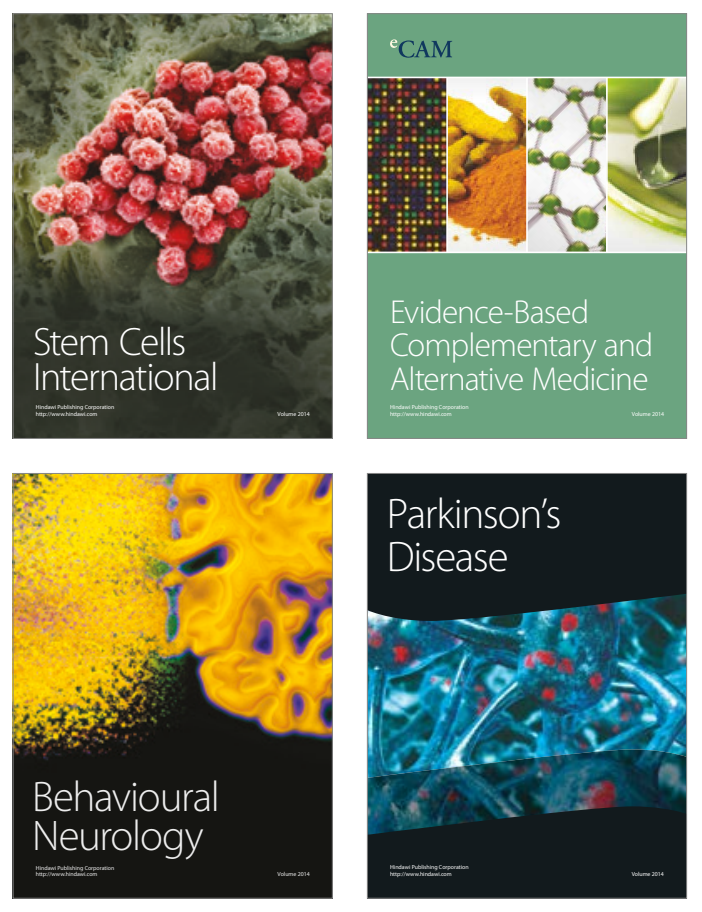
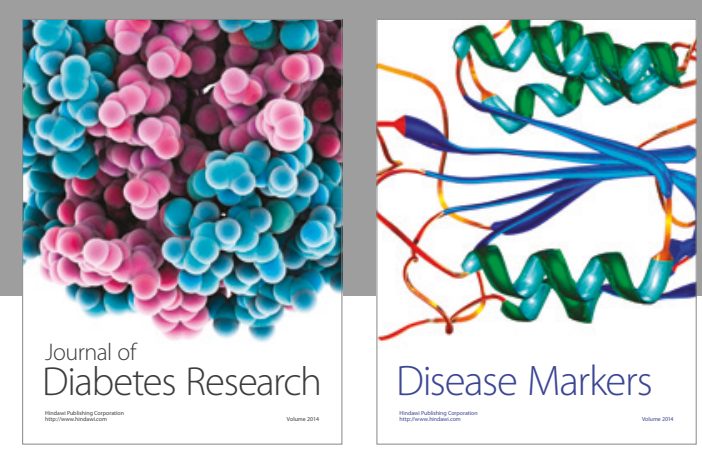

Disease Markers
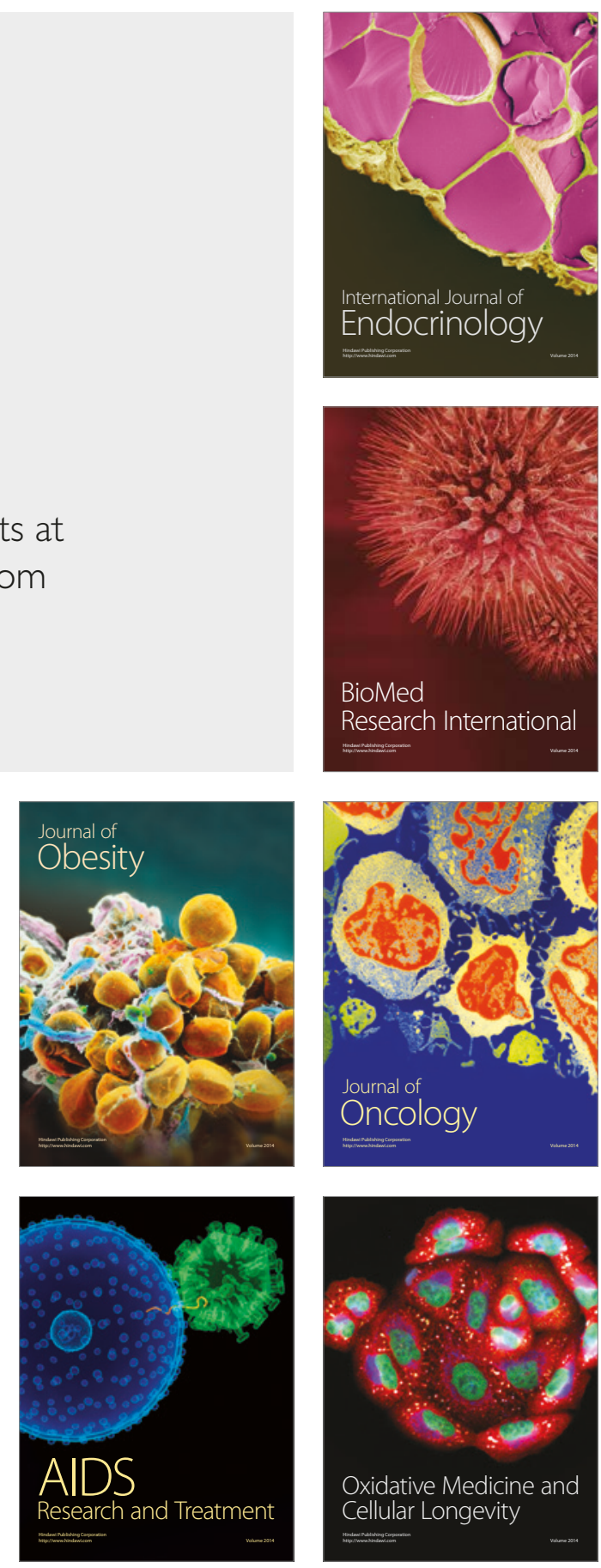